Article

\title{
Broad UV Emission Lines in Type-1 Active Galactic Nuclei: A Note on Spectral Diagnostics and the Excitation Mechanism
}

\author{
Paola Marziani ${ }^{1, *(\mathbb{D}}$, Ascension del Olmo ${ }^{2}{ }^{\circ}$, Jaime Perea ${ }^{2}$, Mauro $\mathrm{D}^{\prime}$ Onofrio ${ }^{3}(\mathbb{C}$ \\ and Swayamtrupta Panda ${ }^{4,5}$ \\ 1 National Institute for Astrophysics (INAF), Astronomical Observatory of Padova, IT-35122 Padova, Italy \\ 2 Instituto de Astrofisíca de Andalucía, IAA-CSIC, Glorieta de la Astronomia s/n, E-18008 Granada, Spain; \\ chony@iaa.es (A.d.O.); jaime@iaa.es (J.P.) \\ 3 Dipartimento di Fisica \& Astronomia “Galileo Galilei”, Università di Padova, IT-35122 Padova, Italy; \\ mauro.donofrio@unipd.it \\ 4 Center for Theoretical Physics (Polish Academy of Sciences), Al. Lotnikó w 32/46, 02-668 Warsaw, Poland; \\ spanda@camk.edu.pl \\ 5 Nicolaus Copernicus Astronomical Center (Polish Academy of Sciences), ul. Bartycka 18, \\ 00-716 Warsaw, Poland \\ * Correspondence: paola.marziani@inaf.it; Tel.: +39-0498293415
}

Received: 9 November 2020; Accepted: 9 December 2020; Published: 18 December 2020

check for updates

\begin{abstract}
This paper reviews several basic emission properties of the UV emission lines observed in the spectra of quasars and type- 1 active galactic nuclei, mainly as a function of the ionization parameter, metallicity, and density of the emitting gas. The analysis exploits a general-purpose $4 \mathrm{D}$ array of the photoionization simulations computed using the code CLOUDY, covering ionization parameter in the range $10^{-4.5}-10^{+1.0}$, hydrogen density $n_{\mathrm{H}} \sim 10^{7}-10^{14} \mathrm{~cm}^{-3}$, metallicity $Z$ between 0.01 and $100 Z_{\odot}$, and column density in the range $10^{21}-10^{23} \mathrm{~cm}^{-2}$. The focus is on the most prominent UV emission lines observed in quasar spectra, namely NV $\lambda 1240$, Sirv $\lambda 1397$, OIV] $\lambda 1402$, CiV $\lambda 1549$, HeII $\lambda 1640$, AliII $\lambda 1860$, SiIII $\lambda 1892$, and CIII] $\lambda 1909$, and on the physical conditions under which electron-ion impact excitation is predicted to be the dominant line producer. Photoionization simulations help constrain the physical interpretation and the domain of applicability of spectral diagnostics derived from measurements of emission line ratios, reputed to be important for estimating the ionization degree, density, and metallicity of the broad line emitting gas, as well as the relative intensity ratios of the doublet or multiplet components relevant for empirical spectral modeling.
\end{abstract}

Keywords: atomic processes; UV spectroscopy; ionized gas; broad line region

\section{Introduction}

Quasar Spectra: Emission from Mildly Ionized Gas

The spectra of active galactic nuclei (AGN) ${ }^{1}$ can be easily recognized by the presence of broad and narrow optical and UV lines emitted by mildly-ionized species over a wide range of ionization potential $\chi$. For an introduction to the AGN spectra and their interpretation in terms of nebular physics, see, e.g., [1-3] and the references therein. Restricting attention to broad lines, type-1 AGN

1 We will use "quasar" as an umbrella term for type-1 AGN (i.e., with broad lines of full-width half-maximum FWHM $\gtrsim 1000 \mathrm{~km} \mathrm{~s}^{-1}$ ) or, whenever specified, to indicate type-1 AGN of high luminosity. 
spectra invariably show the same high ionization (HILs, $\chi \gtrsim 40-50 \mathrm{eV}$ ) and low ionization lines (LILs, $\chi \lesssim 20 \mathrm{eV}$ ). Broad HILs encompass CIV $\lambda 1549$, HeII $\lambda 1640$, and HeII $\lambda 4686$ as representative specimens. Broad LILs include HI Balmer lines $(\mathrm{H} \beta, \mathrm{H} \alpha), \mathrm{MgII} \lambda 2800$, the CaII IR triplet, and FeII features. Additionally, several lines observed in the UV spectra are associated with parent ionic species whose $\chi$ is in between $\approx 20$ and $40 \mathrm{eV}$ (intermediate ionization lines (IILs)). Representative intermediate ionization lines are CIII] $\lambda 1909$, SiIII] $\lambda 1892$, and AlıII $\lambda 1860$ in a blend around $1900 \AA$.

Even if the optical and UV emission lines are the same, their relative intensities and profiles change in a systematic way, what has become known as the quasar main sequence (MS) [4-6]. The trends of the main sequence in turn provide systematic constraints on the physical conditions of the line emitting gas [7-9], as well as on several multifrequency properties, including radio-loudness (see [10] for a recent review). The MS has been instrumental to the interpretation of two important results.

First, a most important trend along the MS involves the prominence of a wind/outflow component in the profiles of the optical and UV lines [7,11-13], whose amplitude is affected by the source Eddington ratio and luminosity [14-16]. The outflow is associated with an excess of blue-shifted HIL emission with respect to $\mathrm{H} \beta$ and other LILs. Attempts to account for it have involved line profile multicomponent modeling, in which a blueshifted excess is added to a symmetric, virialized component emitting most of the LILs (e.g., [7,17,18]).

Second, the view of the physical conditions of broad line emitting gas has changed considerably in the last decade, thanks especially to the results of reverberation mapping and to the realization of the importance of FeII emission [19]. The emitting region radius was found to be a factor of 10 smaller than previously thought $[20,21]$. The smaller distance of the emitting gas from the central continuum source has important implications for the physical conditions of the gas; the conventional view of the broad line region (BLR) as a system of an emitting cloud characterized by typical densities $n_{\mathrm{H}} \sim 10^{9} \mathrm{~cm}^{-3}$ and column density $N_{\mathrm{c}}=10^{23} \mathrm{~cm}^{-2}$ may be only partially valid, as it is unable to account for the strong FeII emission in quasars [22,23]. Higher densities are needed to maintain the ionization parameter, within reasonable limits. It is now accepted that the bulk of the low ionization lines requires high column density, high particle density, low ionization degree, and high metallicity [8,9,24].

In this paper, we focus our attention on the UV rest frame spectrum of type-1 AGN, and we first provide a summary description of the main emission features that are also the main providers of dynamical and diagnostic information on high redshift quasars [25] (Section 2). We then present an overview of the behavior of the most prominent emission lines as a function of the ionization parameter and density with special attention to the emission mechanism and electron-ion collisional excitation, which is expected to be the dominant mechanism for line emission. Computations are summarized in Section 3, and the results are presented in the form of 2D slices of the 4D parameter space $\left(n_{\mathrm{H}}, U, Z, N_{\mathrm{c}}\right)$ in Section 4 . Several applications are briefly discussed in Section 5.

\section{The UV Emission Lines}

The spectral range between $\approx 1230$ and $1950 \AA$ can be subdivided into four regions, each of them associated with a prominent emission blend: (1) the Ly $\alpha+\mathrm{NV}$ blend; (2) the 1400 blend, made by the SiIV doublet and the OIV] $\lambda 1402$ multiplet; (3) the HIL blend CIV + HeII $\lambda 1640$; and (4) the blend at 1900 made predominantly by IILs. The main constituents of the four blends are listed below.

- The $\operatorname{Ly} \alpha+\operatorname{Nv} \lambda 1240$ blend: The $\chi$ of the $\mathrm{NV} \lambda 1240$ parent ionic species $\approx 78 \mathrm{eV}$ is the highest among the line considered here. The $\mathrm{NV} \lambda 1240$ is due to a resonant transition $\left({ }^{2} P_{\frac{3}{2}, \frac{1}{2}}^{\mathrm{o}} \rightarrow{ }^{2} S_{\frac{1}{2}}\right)$ in the lithium isoelectronic configuration;

- The $1400 \AA$ A blend [26]: The SiIV doublet is also a resonant doublet $\left({ }^{2} P_{\frac{3}{2}, \frac{1}{2}}^{o} \rightarrow{ }^{2} S_{\frac{1}{2}}\right.$, from the sodium isoelectronic configuration, $\left.1 s^{2} 2 s^{2} 2 p^{6} 3 s^{1}\right)$. The creation ionization potential of $\mathrm{Si}^{3+}$ is much lower, $\approx 34 \mathrm{eV}$, than the one of $\mathrm{N}^{+4}$. The OIV] $\lambda 1402$ inter-combination multiplet is due to transitions between a ${ }^{4} P$ term and ${ }^{2} P^{o}$ term where the first term is at $0.04785 \mathrm{eV}$ above ground level, with critical densities in the range $n_{\mathrm{c}} \approx 2 \times 10^{10}-6 \times 10^{11} \mathrm{~cm}^{-3}[27,28]$; 
- The CIV+ HeII $\lambda 1640$ blend: The CIV line is a resonant doublet $\left({ }^{2} \mathrm{P}_{\frac{3}{2}, \frac{1}{2}}^{\mathrm{o}} \rightarrow{ }^{2} \mathrm{~S}_{\frac{1}{2}}\right)$ and is again emitted by a transition in the lithium isoelectronic configuration. The parent ionic species has an ionization potential of $\approx 50 \mathrm{eV}$. HeII $\lambda 1640$ is emitted via $3 \mathrm{~d}^{2} \mathrm{D} \rightarrow 2 \mathrm{p}^{2} \mathrm{P}^{\mathrm{o}}$, which corresponds to a transition between two very high energy levels above the ground state (48 and $40 \mathrm{eV})$. The HeII $\lambda 1640$ line is blended with the red side of CIV, and the two lines are often measured together [29].

- The blend at $\lambda 1900 \AA$ is due, in most part, to the AlIII $\lambda 1860$ doublet and to the SiIII] $\lambda 1892$ and CIII] $\lambda 1909$ lines. AlIII is a resonant doublet as CIV $\left({ }^{2} P_{\frac{3}{2}, \frac{1}{2}}^{o} \rightarrow{ }^{2} S_{\frac{1}{2}}\right)$ in the sodium isoelectronic configuration, while SiIII] and CIII] are due to inter-combination transitions $\left({ }^{3} P_{1}^{o} \rightarrow{ }^{1} \mathrm{~S}_{0}\right)$ with widely different critical densities $\left(\approx 2 \times 10^{12} \mathrm{~cm}^{-3}\right.$ and $\approx 3.2 \times 10^{9} \mathrm{~cm}^{-3}$, respectively; [18]). The parent ionic species imply ionization potentials $20 \lesssim \chi_{\mathrm{i}} \lesssim 30 \mathrm{eV}$, intermediate between the ones of the LILs and of the HILs; $\chi_{\mathrm{i}} \gtrsim 40-50 \mathrm{eV}$.

\section{Photoionization Computations}

Photoionization simulations require inputs in terms of several parameters that allow defining the ionization and level populations along with electron temperature and optical thickness as a function of the geometrical depth in a cloud (a slab) of emitting gas. The geometry we assumed is open, plane parallel, meaning that a slab of emitting gas is exposed to a radiation field only on one side. The input provided for a full computation of the emitting gas state, and hence for the prediction of the emission line intensities, includes the following parameters:

- the ionization parameter $U=\frac{\int_{v_{0}}^{\infty} \frac{L_{v}}{h v}}{4 \pi r_{\mathrm{BLR}}^{2} c n_{\mathrm{H}}}=\frac{Q(H)}{4 \pi r_{\mathrm{BLR}}^{2} c n_{\mathrm{H}}}$, where $Q(H)$ is the number of ionizing photons and $r_{\text {BLR }}$ the distance between the continuum source and the line emitting gas, provides the ratio between the hydrogen-ionizing photon and the hydrogen number density;

- the hydrogen density $n_{\mathrm{H}}$;

- $\quad$ the metallicity $Z$;

- the quasar spectral energy distribution (SED);

- the column density $N_{c}$;

- a micro-turbulence parameter [8].

It is a six-dimensional parameter space that is customarily not explored in full in photoionization computations. Here, we will consider trends in $n_{\mathrm{H}}, U, Z, N_{\mathrm{c}}$, assuming zero micro turbulence and a typical AGN SED as parameterized by [30]. The SED is appropriate for luminous type-1 AGN, radiating at a moderate Eddington ratio [31].

The code CLOUDY is designed to model environments that range from the low density limit to thermodynamic equilibrium $[32,33]$. CLOUDY models the ionization, chemical, and thermal state of gas exposed to a radiation field and predicts its emission spectra and physical parameters. In CLOUDY, collisional excitation and radiative processes typical of mildly ionized gases are included, at the expense of the radiation transfer, which is treated via a mean escape probability formalism. Maps are built on arrays of 667 simulations of the CLOUDY 13.05 and 17.02 photoionization models for a given metallicity $Z$ and $N_{c}$, with $n$ and $U$ evaluated at steps of 0.25 dex covering the ranges $7 \leq \log n_{\mathrm{H}} \leq 14 \mathrm{~cm}^{-3}$, $-4.5 \leq \log U \leq 0$. They were repeated for 12 values of the metallicity, in the range $0.01 Z_{\odot}-100 Z_{\odot}$. Three values of $N_{c}$ were considered $\log N_{c}=21,22$, and $23 \mathrm{~cm}^{-2}$. The macro trends described in the isophotal contours representing $\left(U, n_{\mathrm{H}}\right)$ slices of the parameter space and presented in Section 4 were validated by the use of both CLOUDY 13.05 and 17.02 .

\section{Results}

\subsection{Trends with the Ionization Parameter and Density}

The left panels of Figure 1 show the total intensity relative to $\mathrm{H} \beta$ of the NV, SiIV, CIV, and AlIII doublets, for $\log N_{\mathrm{c}}=23\left(\mathrm{~cm}^{-2}\right)$, and solar metallicity, as a function of the ionization parameter 
and hydrogen density. ${ }^{2}$ The four lines show behaviors that are qualitatively similar. The dominant excitation mechanism for optically-thick lines is expected to be provided by electron-ion collisions, a process briefly summarized in Appendix A. To ascertain the range of parameters over which electron-ion collision is the dominant excitation mechanism, we define the ratio:

$$
R_{\mathrm{c}}=\frac{\sum_{j} I_{\mathrm{j}, \mathrm{coll}}}{\sum_{\mathrm{j}} I_{\mathrm{j}}}
$$

where the sum is done for $j=1,2$ for the doublets. Only $j=1$ is considered for HeII $\lambda 1640$, CIII], and SiIII]. The ratio $R_{\mathrm{C}}$ is shown as a function of $n_{\mathrm{H}}$ and $U$, for two metallicity cases, $1 Z_{\odot}$ and $20 Z_{\odot}$ for the various doublets, for HeII $\lambda 1640$, and for the semi-forbidden lines in Figures $1-3$. Figure 1 shows that collisional excitation is by far the dominant mechanism for the formation of the CIV doublet over a very broad range of ionization parameters and density, with $R_{\mathrm{c}}$ becoming significantly less than one for $\log U \lesssim-4$ and $\log U \gtrsim 0.5$. Considering, for example, a very low ionization case $\left(\log U, \log n_{\mathrm{H}}\right)=(-4.0,12)$, the dominant ionization stages of carbon change from triply to singly ionized. At such low $U$, the CIV line is extremely weak, and its emission is sustained by collisional excitation only close to the illuminated face of the cloud. At $\log U \sim-4$, the dominant emission mechanism is provided by continuum pumping.

At high $U$, the collisional excitation remains the main contributor, $R_{\mathrm{c}} \gtrsim 0.5$, but the dominant ionization state is four-times-ionized carbon, making the CIV line weak. The behavior of $R_{\mathrm{c}}$ and of $T_{\mathrm{e}}$ as a function of $U$ is shown in Figure 4. When the radiation field is intense, continuum pumping can be a formation mechanism for the high excitation lines like CIV. At $\log U \gtrsim 0$, continuum pumping accounts for the missing fraction in the line excitation.

At high $n_{\mathrm{H}}, R_{\mathrm{C}}$ can be larger than one if $Z$ is high (regions in yellow / pale green in the isophotal contour maps of Figure 1). The line may be radiatively excited via fluorescent excitation by the incident continuum and then collisionally deexcited. This process tends to thermalize the line and to heat the gas. It is especially relevant at high density and a high ionization parameter.

For the $Z=20 Z_{\odot}$ cases, $R_{\mathrm{c}}$ starts to drop at higher $U$. The parameters $\log U \gtrsim 0$ and $T_{\mathrm{e}} \gtrsim 1 \times 10^{5}$ $\mathrm{K}$ may imply that the photoionization solution is thermally unstable. The higher metal abundances (especially at high density where collisions are most efficient) provides an efficient cooling, lowering $T_{\mathrm{e}}$ and stabilizing the emitting gas against a thermal runaway at high $U$ (Figure 4).

The behavior of $R_{\mathrm{c}}$ for SiIV and NV in the plane $\log U, \log n_{\mathrm{H}}$ is substantially analogous to that of CIV (second row of Figure 1). Collisional excitation ceases to be effective at very high and low values of the ionization parameter for $Z=1 Z \odot$. The main differences are related to the different $\chi$ of the parent ions and to the difference in excitation potential. Given the assumed SED [30] that peaks at a few tens of $\mathrm{eV}$, the higher the ionization potential of any ionic species above $40 \mathrm{eV}$, the fewer the ionizing photons will be. Nv $\lambda 1240$ (top row Figure 1) is the line with the highest $\chi$ and excitation potential and understandably becomes extremely faint at higher $U$ than the other lines: below $\log U \approx-3.25$, it is exceedingly fainter with respect to the intensity of $\mathrm{H} \beta$. In the $Z=20 \mathrm{Z}_{\odot}$ case (rightmost panel of Figure 1, second row), the relative intensity remains high and the collisional excitation the exclusive excitation mechanism up to $\log U \sim 1$. This effect is seen for all the three lines, NV, CIV, and SiIV, emitted by ionic species of higher ionization potential.

2 We included very low levels of relative intensity in Figure 1 and in the following ones showing isophotal contours. However, levels at $\sim 10^{-11}$ of relative intensity are clearly not detectable and also hardly predictable with good precision. An appropriate range of the intensity ratio is $10^{-3} \lesssim$ Line $/ \mathrm{H} \beta \lesssim 10^{3}$. Outside of this range, either the line in consideration or $\mathrm{H} \beta$ would be too faint to be detected with commonly used instruments. 

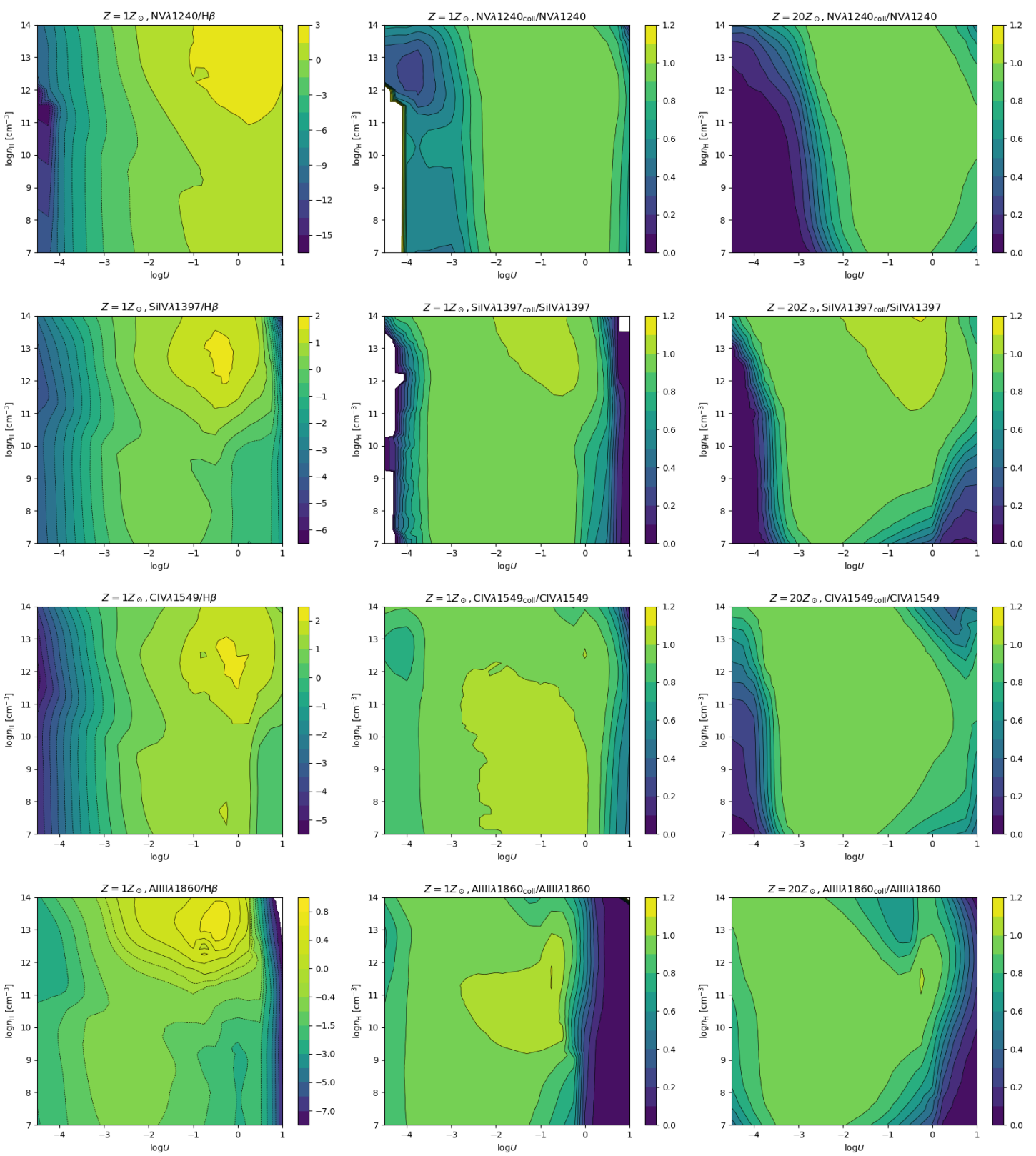

Figure 1. Leftmost panels: the decimal logarithm of the ratio between resonance emission lines and $\mathrm{H} \beta$ as a function of ionization parameter $U$ and hydrogen density $n_{\mathrm{H}}$, from arrays of 667 simulations from CLOUDY 17.02. From top to bottom: NV, SiIV, CIV, AlIII. Middle and right panels: the ratio of collisional excitation emission to the total line emission, again as a function of ionization parameter $U$ and hydrogen density $n_{\mathrm{H}}$. Middle: $Z=1 Z_{\odot}$; right: $Z=20 Z_{\odot}$. 

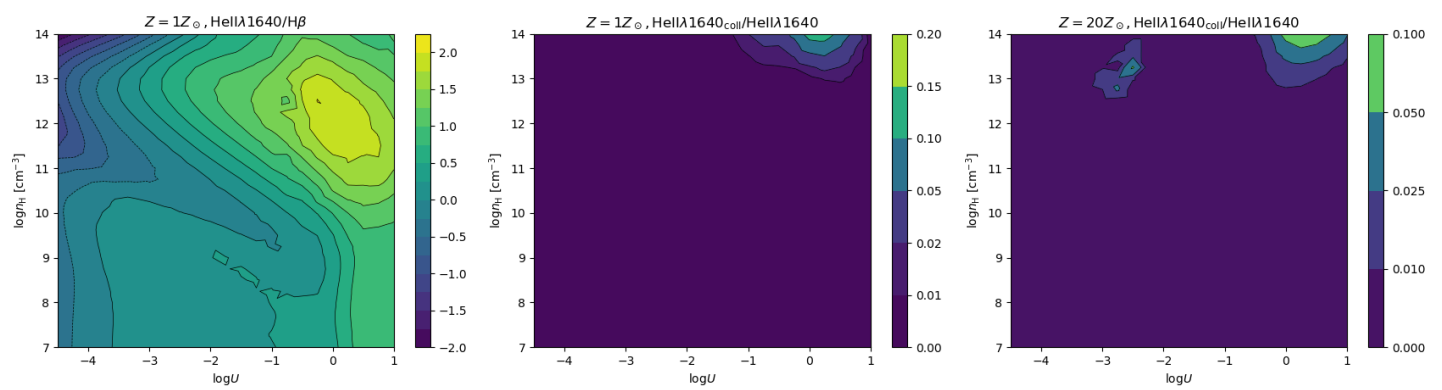

Figure 2. Left panel: the decimal logarithm of the ratio between HeII $\lambda 1640$ and $H \beta$ as a function of ionization parameter $U$ and hydrogen density $n_{\mathrm{H}}$, computed as in the previous figure. Middle and right panels: the ratio of collisional excitation emission to total line emission for the HeII $\lambda 1640$ line, again as a function of ionization parameter $U$ and hydrogen density $n_{\mathrm{H}}$. Left: $Z=1 Z_{\odot}$; right: $Z=20 Z_{\odot}$.
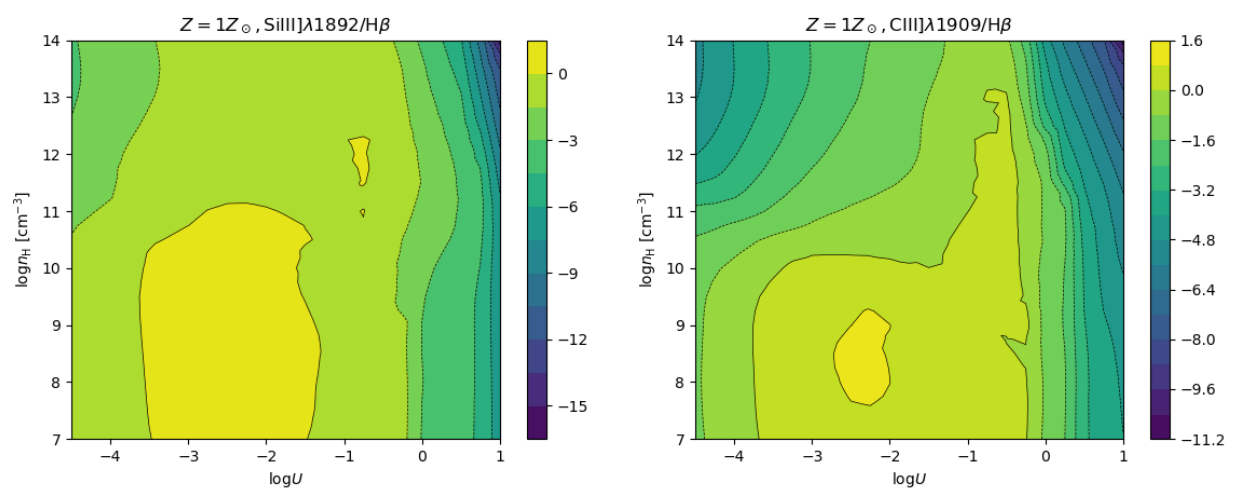

Figure 3. The decimal logarithm of the ratio between intercombination emission lines and $\mathrm{H} \beta$ as a function of ionization parameter $U$ and hydrogen density $n_{\mathrm{H}}$, as in the previous figures. Left: SiIII]/H $\beta$; right: $\mathrm{CIII}] / \mathrm{H} \beta$.
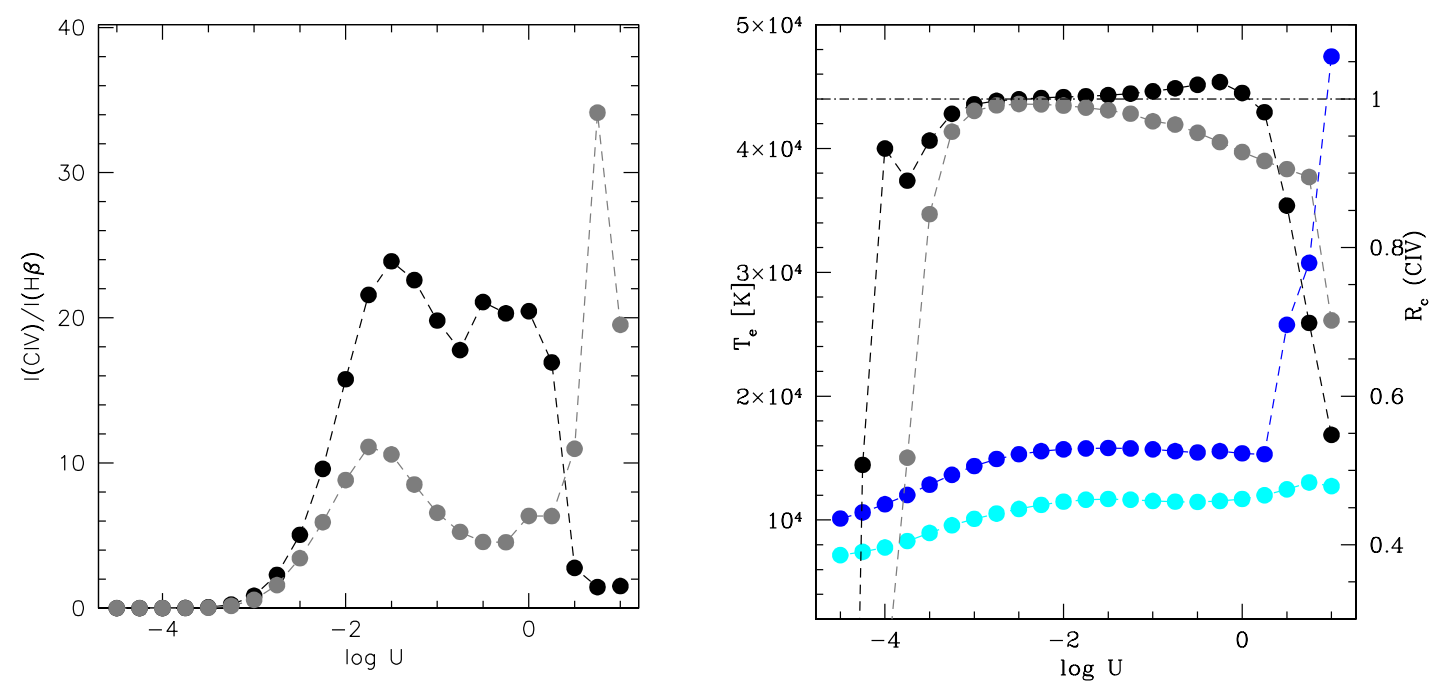

Figure 4. Left panel:the intensity ratio $\mathrm{CIV} / \mathrm{H} \beta$ as a function of the logarithm of the ionization parameter, for $n_{\mathrm{H}}=10^{9} \mathrm{~cm}^{-3}$, for $\mathrm{Z}=1 Z_{\odot}$ (black dots) and $\mathrm{Z}=20 \mathrm{Z}_{\odot}$ (grey dots), assuming $\log N_{\mathrm{c}}=$ $23\left(\mathrm{~cm}^{-2}\right)$. Right: average electron temperature (blue) over the radius of the CIV emitting zone in the gas slab and $R_{\mathrm{c}}$ of CIV (black) as a function of the logarithm of the ionization parameter, for $Z=1 Z_{\odot}$ and the same $N_{\mathrm{c}}$ and $n_{\mathrm{H}}$. Cyan circles show $T_{e}$ and grey ones $R_{\mathrm{c}}$ for $Z=20 Z \odot$. 
The case of the IIL AlIII is essentially analogous (Figure 1, bottom row) to the ones of the HILs, and the same trends are well visible. However, there are important differences. The AlIII line is weaker at lower densities and, at the high densities expected for broad line emission, becomes very weak at $\log U \lesssim 0$, whereas the HILs in Figure 1 remain strong. Collisional excitation remains the dominant excitation mechanism up to ionization parameters $\log U \lesssim-0.5$, much lower than that of the HILs. At $\log U \gtrsim 0$, the AlIII intensity is very low or almost nil. The weakness of the AlIII line at relatively modest $U \sim-0.5$ has important consequences in the interpretation of quasar spectra (briefly summarized in Section 5). In the case of $Z=20 Z_{\odot}$ (rightmost panel of Figure 1 , bottom row), the AlıI behavior is more similar to the ones of the higher ionization lines. At high $n_{\mathrm{H}}\left(\approx 1 \times 10^{12} \mathrm{~cm}^{-3}\right)$, $R_{\mathrm{c}}$ remains $\gtrsim 0.5$ up to $\log U \approx 0.75$.

The computations for HeII $\lambda 1640$ (Figure 2) confirm the expectation of a negligible role of collisional excitation, although at very high density $\left(\log n_{\mathrm{H}} \sim 10^{13}-10^{14} \mathrm{~cm}^{-3}\right)$ and relatively high $U$ $(\log U \sim-0)$, the contribution of collisional excitation can apparently reach $R_{\mathrm{C}} \approx 0.1-0.2$.

The intensity of the SiIII] inter-combination line (an IIL) with respect to $\mathrm{H} \beta$ shows a clear dependence on density (Figure 3, leftmost panel) for low and moderate values of $U$. While the line is emitted most efficiently at relatively low density $\left(n_{\mathrm{H}} \sim 10^{9-10} \mathrm{~cm}^{-3}\right)$, the line remains strong also at high density $\left(n_{\mathrm{H}} \sim 10^{12} \mathrm{~cm}^{-3}\right)$. On the high ionization side of Figure 3 , the line becomes undetectable for $\log U \gtrsim 0$. The CIII] behavior in the plane $\left(U, n_{\mathrm{H}}\right)$ is influenced by the much lower critical density at low $U$; the area of most efficient emission is centered at $\log n_{\mathrm{H}} \sim 10^{8}$. In the region of Figure 3 expected for the low ionization part of the BLR, the CIII] intensity should be $\lesssim 0.1 \mathrm{H} \beta$, barely detectable if at all [18]. Note however the parameter region at high ionization $(\log U \sim-0.5)$ extended up to high density where the CIII] line remains strong with respect to $\mathrm{H} \beta$.

Collisional excitation is conventionally expected to be dominant for both CIII] and SiIII]: CLOUDY 13.05 results imply $R_{\mathrm{C}} \approx 1$, although CLOUDY 17.02 predicts $R_{\mathrm{C}} \approx 0.5$ for $Z=1 Z_{\odot}$ and $Z=20 Z_{\odot}$ in the parameter region where the lines are emitted efficiently. The lower values obtained by CLOUDY 17.02 are likely to be associated with the modest optical depth of the two lines, and to a different treatment of collisional excitation for some inter-combination lines.

\subsection{Dependence on Metallicity}

Figure 5 shows the behavior as a function of $\log Z$ for the ratio $R_{\mathrm{c}}$ for a typical low ionization $(-2.5,12)$ and high ionization case $(0,9)$, meant to be representative of the virialized, low ionization and of the high ionization outflow sub-regions revealed by the emission line profiles. Above solar metallicity, collisional excitation accounts for most $\left(R_{\mathrm{c}} \gtrsim 0.9\right)$ or all line emission save in the case of $\mathrm{NV}$ at low ionization. In the high ionization case, AlıII shows $R_{\mathrm{C}}$ values that are less than 0.5 up to $Z \approx 5 Z$. In this domain, however, the AlıI line is very weak. Since $R_{\mathrm{C}}$ is related to the line intensity, in the sense that $R_{\mathrm{c}}$ differs significantly from one only if the line is weak with respect to $\mathrm{H} \beta$, Figure 5 implies a dependence on $Z$ of the AlıII/H $\beta$ and $\mathrm{NV} / \mathrm{H} \beta$ intensity ratio in the high ionization case. Intensity ratios involving AlıII and Nv have been indeed used as Z diagnostics for quasars [34-36]. 

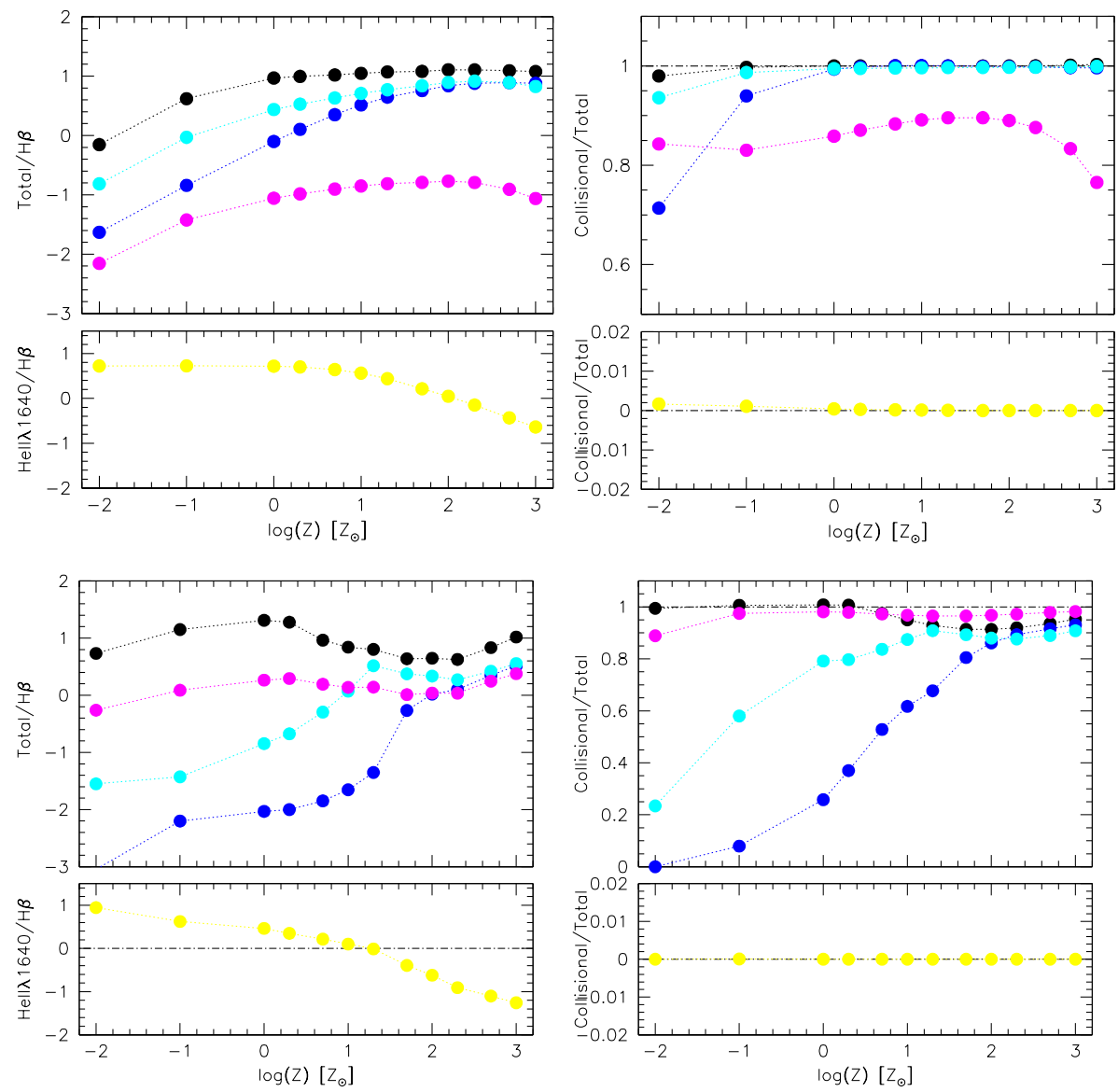

Figure 5. Left panels: the decimal logarithm of the intensity of CIV relative to $\mathrm{H} \beta$ (black circles, $\bullet$ ), AliII (blue, •), Sirv (cyan, •), and NV (magenta, $\bullet$ ) as a function of metallicity Z in solar units; the lower panel shows the same trend for the HeII $\lambda 1640$ line (yellow, $\odot$ ). Right panels: the dependence of the ratio collisional emission over total line intensity $R_{\mathrm{c}}$ for CIV on metallicity, with the same meaning of the symbols. Top panel: low ionization case; bottom: high ionization case.

\subsection{Dependence on Column Density and on Optical Depth}

The CIV and AlIII intensities relative to $\mathrm{H} \beta$ and the ratio $R_{\mathrm{c}}$ as a function of column density $N_{\mathrm{c}}$ are reported in Table 1 , assuming $Z=1 Z_{\odot}$. In the high ionization case $(0,9)$, CIV remains strong at all $N_{\mathrm{c}}$ values, and the contribution of collisional excitation increases steadily with $N_{\mathrm{c}}$. The AlIII doublet is predicted to be always faint in this case. In the low ionization case $(-2.5,12)$, a significant decrease in the collisional excitation is seen only for $\log N_{\mathrm{c}}=21\left(\mathrm{~cm}^{-2}\right)$. Values of $N_{\mathrm{c}}$ much higher than $\log N_{\mathrm{c}}=23\left(\mathrm{~cm}^{-2}\right)$ are likely for the low ionization part of the broad line region [8,9], but it is expected that both CIV and AlIII will remain strong, with $R_{\mathrm{c}}$ close to unity.

Table 1. Intensity and collisional excitation as a function of column density.

\begin{tabular}{cccccc}
\hline $\log N_{\mathbf{c}}$ & Parameter & \multicolumn{2}{c}{ High } & \multicolumn{2}{c}{ Low } \\
& & CIV & AlIII & CIV & AlIII \\
\hline 23 & $\mathrm{I} / \mathrm{I}(\mathrm{H} \beta)$ & 20.45 & 0.01 & 9.27 & 0.79 \\
23 & $R_{\mathrm{c}}$ & 1.01 & 0.26 & 1.00 & 0.99 \\
22 & $\mathrm{I} / \mathrm{I}(\mathrm{H} \beta)$ & 3.91 & $\ldots$ & 9.46 & 0.81 \\
22 & $R_{\mathrm{C}}$ & 0.90 & $\ldots$ & 1.00 & 0.99 \\
21 & $\mathrm{I} / \mathrm{I}(\mathrm{H} \beta)$ & 6.04 & $\ldots$ & 14.69 & 1.11 \\
21 & $R_{\mathrm{c}}$ & 0.34 & $\ldots$ & 0.46 & 0.58 \\
\hline
\end{tabular}


The ionization structure of the line emitting gas slab is such that the highest ionization occurs close to the illuminated face of the slab (Figure 6; the slab column density has been set to a large value to analyze the ionization degree over a large range of depths) and that the highest ionization lines are also emitted more efficiently closer to the illuminated face than other lines (cf. NV and AliII). The AliII and SiIII] lines can be efficiently emitted in the outer part of the fully ionized zone (FIZ), where there is the highest fraction of the parent ionic species. If the column density decreases, the slab may become optically thin in the HI ionizing continuum, and the AlIII and SiIII] lines may not be emitted at all or at least greatly reduced. In the low ionization case, the region where the parent ionization species is present may be progressively eaten away as $N_{\mathrm{c}}$ decreases. In the high ionization case $(0,9)$, we expect minimal emission from SiIII], AlIII, and SiIV, as there is only a tiny region at the end of the FIZ that makes possible efficient emission of these lines (right panel of Figure 6).
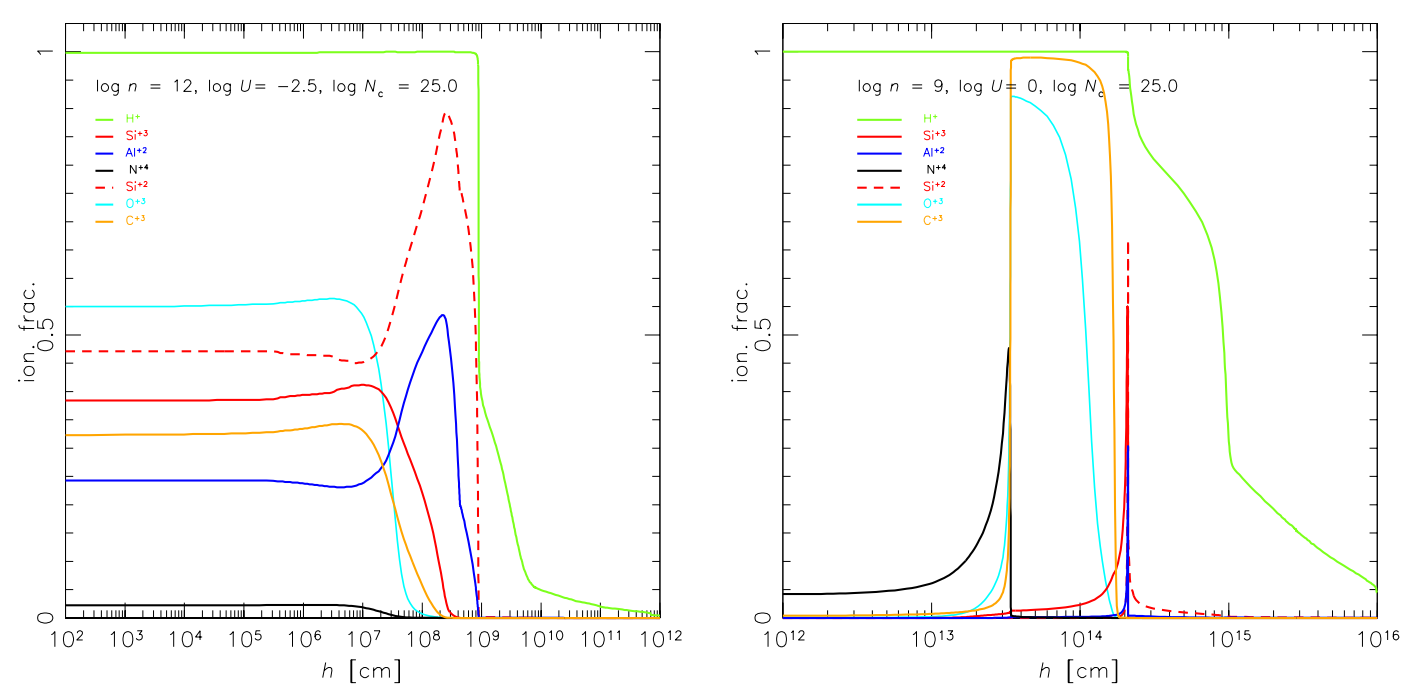

Figure 6. Ionization degree as a function of depth $h$ in the illuminated slab of emitting gas, exposed from the left side to the radiation field. Left panel: low ionization case; right panel: high ionization case.

Electron-ion collisions are expected to be the dominant contributor to optically thick resonance lines. For $\log N_{\mathrm{c}}=23\left(\mathrm{~cm}^{-2}\right)$ and $Z \gtrsim 1 Z_{\odot}$, all lines considered here are optically thick, with the minimum $\tau$ for the inter-combination lines. The optical depth is however dependent on $N_{\mathrm{c}}$ (low at low $N_{\mathrm{c}}$ ), as well as on the ionization state. Figure 6 implies that for $\mathrm{CIV}$, the depth is much higher at high ionization, as the column density of the parent ionic species is much larger than in the case of low ionization. This remains true up to the point where the $N_{c}$ of the parent ionic species remains large. At high ionization, the lines become less optically thick (Figure 7), and an important contribution is provided by continuum pumping. The optical depth of the lines also increases with $Z$, being just $\tau \sim 10-10^{2}$ for $Z \approx 0.01 Z_{\odot}$, and reaching $\tau \gtrsim 10^{7}$ for $Z \gtrsim 1 Z_{\odot}$ in the high ionization case and $\tau \gtrsim 10^{3}-10^{4}$ in the low ionization case. 


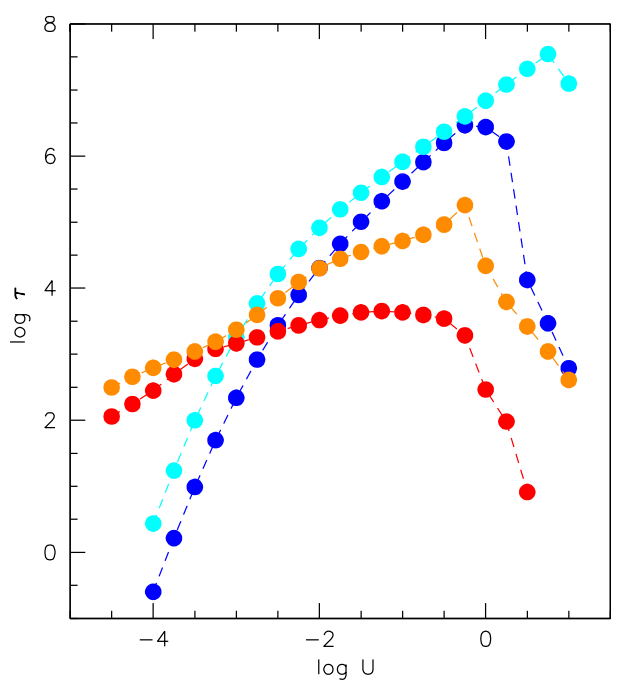

Figure 7. Line optical depth $\tau$ of CIV (blue and cyan) and AliII (red and orange) as a function of the ionization parameter $U$, for $Z=1 Z_{\odot}$ (blue and red) and $20 Z_{\odot}$ (cyan and orange), assuming $\log n_{\mathrm{H}}=9 \mathrm{~cm}^{-3}$.

\section{Discussion}

\subsection{BLR Physical Conditions Along the Quasar Main Sequence}

Several intensity ratios introduce more or less well-defined constraints on the ionization, metallicity, and density of the emitting gas, as summarized in Table 2.

Table 2. Spectral diagnostics.

\begin{tabular}{cc}
\hline Intensity Ratio & Sensitive to \\
\hline SiIV+ OIV] $\lambda 1402 /$ SiIII] & ionization \\
SI II $\lambda 1816 /$ SiIII] & \\
CIV $/$ SiIV + OIV] $\lambda 1402)$ & \\
NV $/$ CIV & \\
CIV $/$ HeII $\lambda 1640$ & metallicity \\
(SiIV + OIV] $\lambda 1402) /$ HeII $\lambda 1640$ & \\
NV $/$ HeII $\lambda 1640$ & \\
AlIII $/$ SiIII $]$ & \\
SiIII] $/$ CIII $]$ & density \\
CIV $/$ AlIII & \\
CIV $/$ SiIII $]$ & ionization * \\
\hline
\end{tabular}

^ Dependent on metallicity.

These intensity ratios have been used in several works in the last 20 years $[7,18,25,34,36-41]$ to constrain the physical conditions within the BLR. The difference in critical density $n_{\mathrm{c}}$ of SiIII] and CIII] allowed for the definition of diagnostic intensity ratios such as CIII]/SiIII] and SiIII]/AlIII sensitive to density $[18,40]$. In addition, the measurements in two intervals of radial velocity, one close to the rest frame and one shifted to the blue (by several hundreds $/ 1000 \mathrm{~km} \mathrm{~s}^{-1}$ ), suggest widely different physical conditions: close to the rest frame, high density and low ionization; in the spectral range shifted to the blue, high ionization and uncertain density.

The spectral diagnostics associated with the photoionization computations allowed for the interpretation of several empirical trends along the quasar MS. For example, within the 1900 blend, we observe a systematic decrease in the prominence of the CIII] line with respect to SiIII] and AlIII, passing from spectra with broader lines and weak FeII to narrower lines and stronger FeII [42]. Sulentic and collaborators distinguished between Population A and B, where Population B includes 
the sources with broader lines (FWHM H $\beta \gtrsim 4000 \mathrm{~km} \mathrm{~s}^{-1}$ [5,43]). The two populations are separated by a $L / L_{\text {Edd }}$ divide at $\approx 0.1-0.3$. In Population $B$, we see evidence of higher ionization and a range of densities [7]. Most Population A sources are characterized by moderate or strong FeII optical emission and with evidence of low ionization (weak CIV HILs, save NV). The CIII] is weak, and the density-sensitive diagnostics suggests high density $\left(n_{\mathrm{H}} \gtrsim 10^{11} \mathrm{~cm}^{-3}\right)$. These constraints in turn provide support particular dynamical and structural scenarios (e.g., [44]).

\subsection{BLR Radius}

The inversion of the equation defining the ionization parameter (Equation (3)) provides a measure of the emitting region radius $r_{\text {BLR }}$ once the ionizing photon density i.e., the product $U n_{H}$, is known. The photon flux can be estimated with good precision from the combination of the diagnostic line intensity ratios listed above: ideally, a pair of ratios such as AlIII/SiIII] (for density) and SiIV/SiIII] (for ionization) should be sufficient, as the lines representing the observed values in the simulation plane $\log U, \log n_{\mathrm{H}}$ should cross at a single point. This has been shown to be the case, although, to improve the reliability of the estimate, several line ratios are used $[18,25,40]$. The photoionization method remains largely unapplied to date. However, with the increasing availability of high S/N ratio spectra thanks to ongoing and forthcoming optical surveys, the method may find widespread application up to relatively high redshifts, $z \lesssim 4$.

\subsection{Diagnostic Ratios to Estimate Metallicity Content within the BLR}

The ratio of two collisionally excited lines at frequencies $v_{0}$ and $v_{1}$ can be written [45] as:

$$
\frac{j_{X^{\mathrm{i}}, \text { coll }}}{j_{\mathrm{Yi}^{\mathrm{j}}, \mathrm{coll}}} \propto\left(\frac{n_{\mathrm{X}_{\mathrm{i}}}}{n_{\mathrm{Y}_{\mathrm{j}}}}\right)^{\kappa} \exp \left(-\frac{h\left(v_{0}-v_{1}\right)}{k T_{\mathrm{e}}}\right)
$$

where $\kappa=1,2$ in the high and low density case, respectively. This means that hydrogen density and $Z$ are constant; the dominant parameter affecting the intensity ratios of the different lines is electron temperature $T_{\mathrm{e}}$; and the ionic fractions can be converted to elemental abundances via an ionic correction mainly dependent on the AGN spectral energy distribution. The ratios of collisionally excited lines are expected to be very effective in the estimation of $Z$ [45]. On the other hand, the ratios involving HeII $\lambda 1640$ take advantage of the independence on $Z$ of the HeII $\lambda 1640$ line. Figure 8 shows the clear dependence of the ratios CIV/HeII $\lambda 1640$ and SiIV+ OIV] $\lambda 1402 / H e I I \lambda 1640$ on $Z$ for a wide range of ionization parameters. The HeII Ly $\alpha$ line at $304 \AA$ ionizes hydrogen atoms before being scattered many times, so that scattering of HeII Ly $\alpha$ cannot sustain a population of electrons at the level $n=2$ of ionized helium (unlike the case of hydrogen, where Ly $\alpha$ is supposed to be scattered ad infinitum). The HeII $\lambda 1640$ line is therefore produced almost exclusively by recombination, and no collisional excitation from level $n=2$ or radiative transfer effects are expected (unlike the case of the H Balmer lines). The prediction of the HeII $\lambda 1640$ line follows, at least in principle, Case B of recombination theory [3], and it is relatively simple once the electron temperature and the density are known by assumption or computation. Use of HeII $\lambda 1640$ should yield robust $Z$ estimates. 

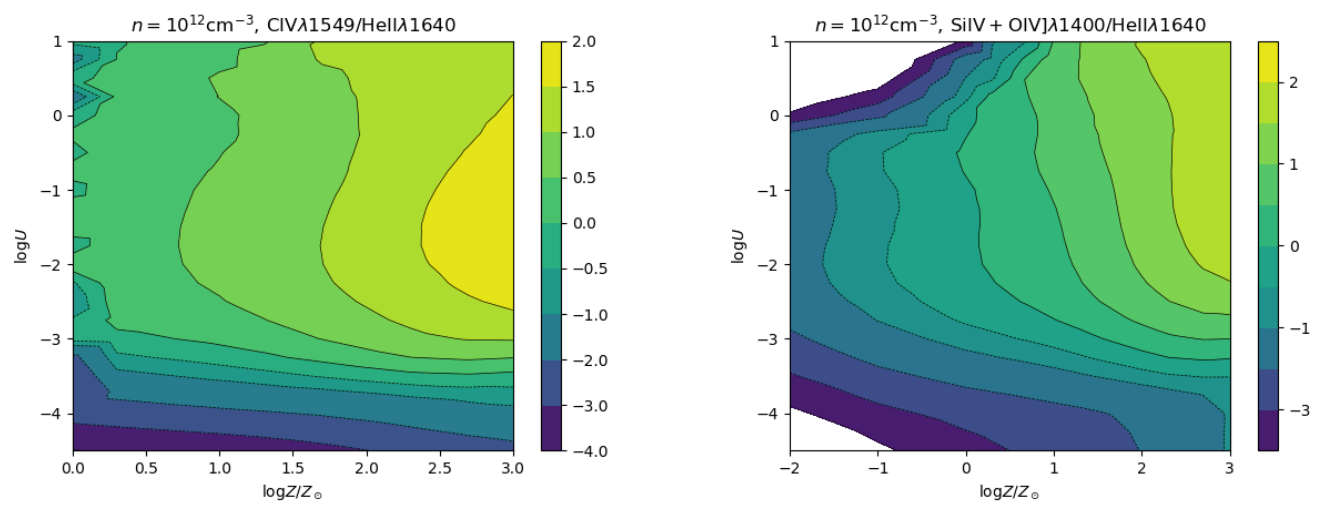

Figure 8. Dependence of the ratios CIV/HeII $\lambda 1640$ and SiIV+ OIV] $\lambda 1402 /$ HeII $\lambda 1640$ (in log) on Z and $U$.

\subsection{Feedback Effects of Accreting Black Holes}

The line emissivity of a collisionally excited line per unit volume can be written as:

$$
j=h v q_{\mathrm{ij}} n_{\mathrm{e}} n_{\mathrm{i}}
$$

where $n_{\mathrm{e}}$ is the electron density and $n_{\mathrm{I}}$ the number density of the parent ionic species at the lower level associated with the transition. The total line luminosity over an emitting volume is:

$$
L=\int_{V} j f_{\mathrm{f}} d V,
$$

where $f_{\mathrm{f}}$ is the filling factor of the emitting gas. The CIV line luminosity can be connected to the mass of outflowing ionized gas $M_{\text {out }}^{\text {ion }}$ and to the mass outflow rate at a distance $r(1 \mathrm{pc}$,$) which can be written,$ if the flow is confined to a solid angle of $\Omega$ [46], as:

$$
\dot{M}_{\text {out }}^{\text {ion }}=\rho \Omega r^{2} v=\frac{M_{\text {out }}^{\text {ion }}}{V} \Omega r^{2} v \approx 15 L_{45} v_{5000} r_{1}^{-1} n_{9}^{-1} \mathrm{M}_{\odot} \mathrm{yr}^{-1}
$$

under the assumption of constant density $\left(n_{9}\right.$ is in units of $\left.10^{9} \mathrm{~cm}^{-3}\right)$. The metallicity is scaled to $10 Z_{\odot}$, appropriate for highly accreting quasars detected at high redshift $[36,41,47,48]$. The corresponding outflow kinetic power, with outflow $v$ in units of $5000 \mathrm{~km} \mathrm{~s}^{-1}$, is:

$$
\dot{\epsilon}=\frac{1}{2} \dot{M}_{\mathrm{out}}^{\mathrm{ion}} v^{2} \approx 1.2 \times 10^{44} L_{45} v_{5000}^{3} r_{1}^{-1} \mathrm{erg} \mathrm{s}^{-1} .
$$

These relations are helpful to measure the dynamical properties of the outflow originating from the accretion disk wind and escaping into the circumnuclear region of the host galaxy. The diagrams of Figures 1 and 3 help to identify the region in the parameter space where the lines are expected to be strong and collisionally excited. In the case of a quasar wind, $U$ cannot be extremely high to account for the cases in which CIV emission is very strong. The assumption of collisional excitation, whenever appropriate, as the dominant excitation mechanism makes it easier to compute the wind dynamical parameters for several emission lines.

It is also interesting to note that the IIL AlIII line, unlike the HILs considered here, is weak unless density is relatively high and the ionization parameter low. The AliI line, even if a resonance line, is more efficiently emitted by the dense gas in the low ionization, virialized part of the BLR, and its emission may not be strong in the quasar high ionization outflows. Broad absorption lines in AliII are detected, but much less frequently than for CIV [49]. The privileged emission in the low ionization, virialized part of the BLR is consistent with the possibility of using the AlIII width as a viral broadening estimator for high redshift quasars [50]. 


\subsection{Applications to Empirical Line Profile Modeling: Intensity Ratios of the Doublet Components}

The issue of the intensity ratios of the doublet components is relevant to the spectral analysis of high- $z$ quasars or low- $z$ type-1 AGNs observed from spaceborne observatories [51]. As the lines originate from the transition between the same spectroscopic terms, we would expect that, in the case of optically thin line emission, the ratio is equal to the ratio of the statistical weight of each level, i.e., $g_{j} / g_{i}=\left(2 J_{j}+1\right) /\left(2 J_{i}+1\right)$, implying a maximum ratio of two for the ratio between the $J=\frac{3}{2}$ and the $J=\frac{1}{2}$ components of the doublet, $\mathrm{I}\left({ }^{2} P_{\frac{3}{2}}^{o} \rightarrow{ }^{2} S_{\frac{1}{2}}\right) / \mathrm{I}\left({ }^{2} P_{\frac{1}{2}}^{o} \rightarrow{ }^{2} S_{\frac{1}{2}}\right)$. The UV emission lines emitted in conditions of moderate to high density and high $N_{c}$ are never fully optically thin. At large optical depths, collisional excitation dominates, and the doublet becomes thermalized: the lines of the doublet should be of equal intensity. This second condition is also not fully realistic: we cannot receive photons from a region of $\tau \rightarrow \infty$. As a matter of fact, the ratio between the AlitI lines $\mathrm{I}\left({ }^{2} P_{\frac{3}{2}}^{o} \rightarrow{ }^{2} S_{\frac{1}{2}}\right) / \mathrm{I}\left({ }^{2} P_{\frac{1}{2}}^{o} \rightarrow{ }^{2} S_{\frac{1}{2}}\right)$ is found to be $\approx 1.25$ [52]. This corresponds to very high densities in the CLOUDY simulation (Figure 9), supported by several lines of evidence for the low ionization part of the $\operatorname{BLR}[7,53,54]$. According to the photoionization simulations, a value of $\approx 1.3$ could be appropriate for both AliıI and SiIV.
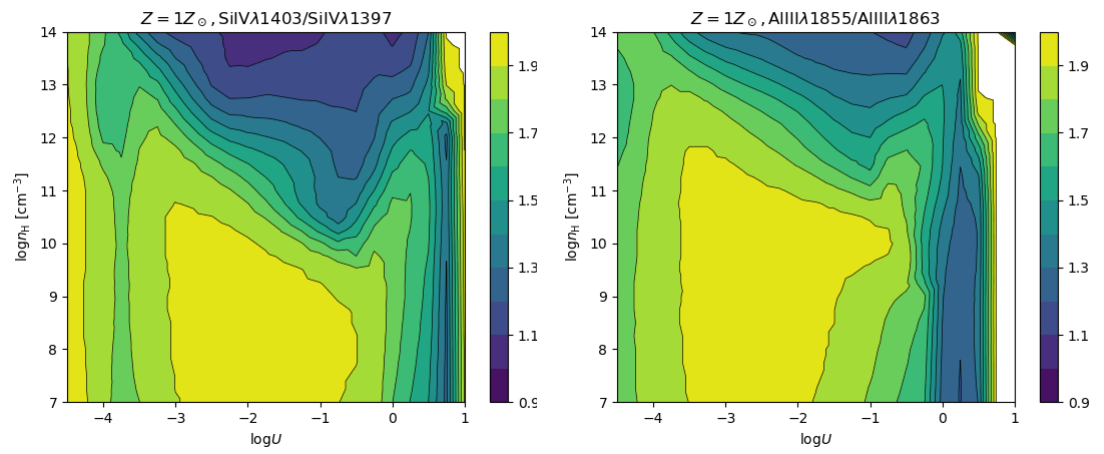

Figure 9. Intensity ratios of the $\mathrm{I}\left({ }^{2} P_{\frac{3}{2}}^{o} \rightarrow{ }^{2} S_{\frac{1}{2}}\right) / \mathrm{I}\left({ }^{2} P_{\frac{1}{2}}^{o} \rightarrow{ }^{2} S_{\frac{1}{2}}\right)$ doublet components for SiIV (left panel) and AlIII (right), as a function of $U$ and $n_{\mathrm{H}}$, for solar metallicity.

\subsection{Applications to Empirical Line Profile Modeling: The Case of OIv]ג1402}

The four blends considered in this paper offer the main diagnostic tools for the physical condition of the BLR emitting gas. In the past, insufficient data quality has often prevented the full exploitation of the UV spectral data. More recently, the quasar MS has offered the possibility to consider each quasar within a trend, which is fairly well defined empirically, but not yet fully connected to physical parameters. In order to achieve this goal, the blends need to analyzed starting from considerations on the physics of line formation. A discussion on the $1900 \AA$ blend can be found in [41], on the 1550 CIV + HeII $\lambda 1640$ blend in [7], and on the NV + Ly $\alpha$ in [34,39]. Here, we focus on the analysis of the OIV] $\lambda 1402+$ SiIV blend, which has been widely used, in combination with other lines, as a $Z$ estimator.

The OIV] $\lambda 1402$ multiplet has been considered as a diagnostic of electron density and temperature [55]. In the context of AGNs, OIV] 11402 is severely blended with SiIV, and the width of the emission lines makes it impossible to distinguish between their relative contributions. CLOUDY includes five lines [56], corresponding to the transitions listed in Table 3. 
Table 3. OIV] $\lambda 1402$ line identification.

\begin{tabular}{ccccc}
\hline EC $^{*}$ & Term & EC $^{*}$ & Term & $\lambda(\AA){ }^{\star}$ \\
\hline $2 s^{2} 2 p$ & ${ }^{2} P_{\frac{1}{2}}^{\mathrm{O}}$ & $2 s 2 p^{2}$ & ${ }^{4} P_{\frac{3}{2}}$ & 1397.2 \\
$2 s^{2} 2 p$ & ${ }^{2} P_{\frac{1}{2}}^{\mathrm{O}}$ & $2 s 2 p^{2}$ & ${ }^{4} P_{\frac{1}{2}}$ & 1399.8 \\
$2 s^{2} 2 p$ & ${ }^{2} P_{\frac{3}{2}}^{\mathrm{O}}$ & $2 s 2 p^{2}$ & ${ }^{4} P_{\frac{5}{2}}$ & 1401.2 \\
$2 s^{2} 2 p$ & ${ }^{2} P_{\frac{3}{2}}^{\mathrm{O}}$ & $2 s 2 p^{2}$ & ${ }^{4} P_{\frac{3}{2}}$ & 1404.8 \\
$2 s^{2} 2 p$ & ${ }^{2} P_{\frac{3}{2}}^{\mathrm{O}}$ & $2 s 2 p^{2}$ & ${ }^{4} P_{\frac{1}{2}}$ & 1407.4 \\
\hline *lectronic configuration. ${ }^{*}$ Line wavelengths from [28].
\end{tabular}

We consider three different cases in terms of the ionization parameter. The first case (top panel of Figure 10) corresponds to the high ionization solution derived for the emission expected from quasar BLR outflows; the second case (middle panel of Figure 10) represents a higher ionization case; the third case is the low ionization, high density case with the parameters expected for the virialized, low ionization part of the BLR. The first case is the one where OIV] $\lambda 1402$ emission is most efficient. The third case, because of the $n_{\mathrm{H}}$ much higher than the critical density of the OIV] $\lambda 1402$ transitions, implies very weak OIV] $\lambda 1402$ emission $(\sim 0.05$ the intensity of $\mathrm{H} \beta)$. The point here is that, even if the relative intensity of the components is affected by the different physical conditions, the profile of their sum remains stable: the peak of emission occurs at $1402.25 \pm 0.10 \AA$ and the FWHM at $\approx 4720 \mathrm{~km} \mathrm{~s}^{-1}$ without any significant difference for the three cases. The $\lambda 1401$ line, with a relatively high spontaneous transition probability for an intercombination line $\left(A \approx 1470 \mathrm{~s}^{-1}\right)$ and a large statistical weight within the multiplet, remains the dominant contributor to the multiplet.

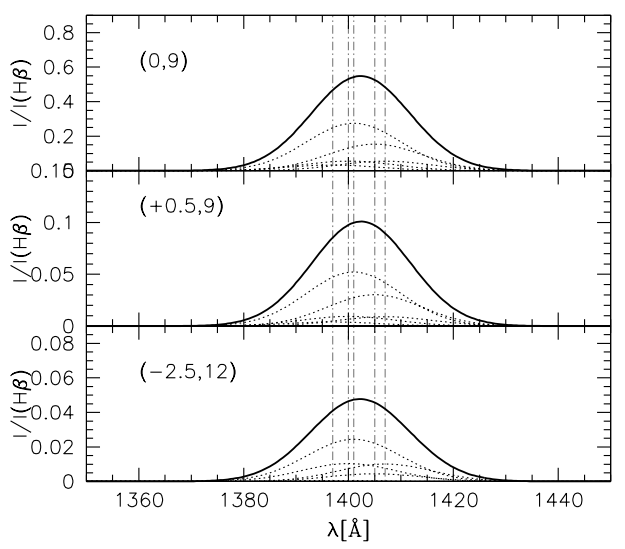

Figure 10. Composite OIV] $\lambda 1402$ profile (thick line), with the individual components of the multiplet as in Table 3, for three case of $\log U, \log n_{\mathrm{H}}$. The ordinate is OIV] $\lambda 1402$ intensity normalized to $\mathrm{H} \beta$ intensity. Individual line dispersion has been set at $9 \AA$, corresponding to an FWHM $\approx 4500 \mathrm{~km} \mathrm{~s}^{-1}$.

The density expected for the low ionization parts of the line emitting regions is $\sim 10^{12}-10^{13} \mathrm{~cm}^{-3}$, at least one order of magnitude above the OIV] $\lambda 1402$ critical densities. Figure 11 shows that the contribution of OIV] $\lambda 1402$ to the emission from the low ionization part of the BLR $(\log U \sim-2.5)$ should be negligible, although the OIV] $\lambda 1402$ line can be prominent with respect to $\mathrm{H} \beta$ at high ionization ( $\log U \sim-0)$ even if $\log n_{\mathrm{H}} \sim 10^{12} \mathrm{~cm}^{-3}$. 

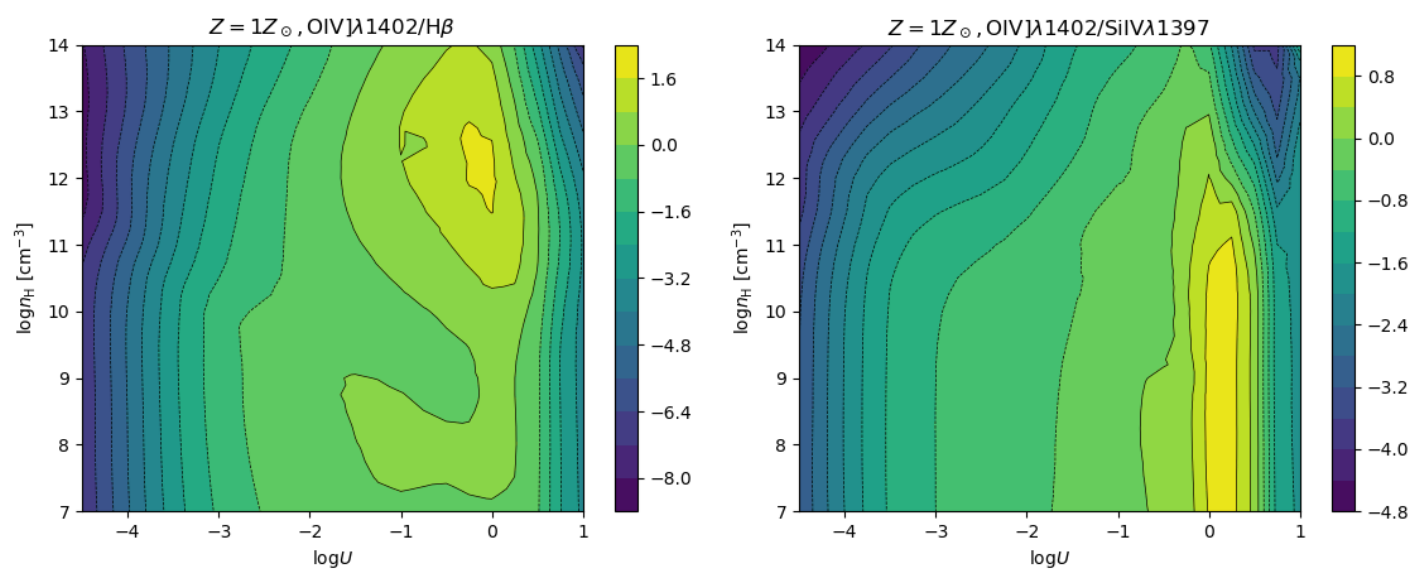

Figure 11. Decimal logarithm of the ratio between the total OIV] $\lambda 1402$ multiplet emission and $\mathrm{H} \beta$ (left) and the total doublet SiIV $\lambda 1397$ emission (right), as a function of ionization parameter $U$ and hydrogen density $n_{\mathrm{H}}$.

\section{Summary and Conclusions}

The prominent "metal" emission lines observed in the UV spectrum of quasars can be accounted for by collisional excitation over a wide range of physical conditions in density, the ionization parameter, column density $\left(N_{\mathrm{c}} \gtrsim 10^{22} \mathrm{~cm}^{-2}\right)$, and metallicity. For example, this is true for the CIV line over the full parameter range $\left(U \sim 10^{-4.5}-10^{+1.0}\right.$, hydrogen density $\left.n_{\mathrm{H}} \sim 10^{7}-10^{14} \mathrm{~cm}^{-3}\right)$, at $Z \gtrsim 1 Z_{\odot}$. Connecting the line intensity ratios to physical parameters requires knowledge of the ionic stage distribution for each element, the consideration of possible differences in optical depth effects, etc. This is provided by the comparison between the intensity predicted by the simulations and the observed UV line intensities. Several helpful constraints can be obtained on the physical conditions of the line emitting region, including density, ionization, and chemical composition. UV line ratios can provide an estimate of the ionizing photon flux and hence a photoionization radius. Its estimate through UV lines directly affected by the ionizing continuum overcomes some of the difficulties of the early photoionization estimates of the BLR based on Balmer lines [57]. The photoionization radius is a key ingredient for more accurate black hole mass estimates at high $z$. In addition, relying on the assumption of collisional excitation permits relatively straightforward estimates of the parameters that are associated with the quasar feedback and are believed to be extremely relevant for the quasar host galaxy evolution [58].

Author Contributions: P.M. wrote the review paper, and P.M. and S.P. performed the photoionization computations. A.d.O., M.D. and S.P. contributed with a critical reading and suggestions. J.P. contributed with support for the photoionization computations. All authors read and agreed to the published version of the manuscript.

Funding: A.d.O. and J.P. acknowledge financial support from the State Agency for Research of the Spanish MCIU through the Center of Excellence Severo Ochoa award for the Instituto de Astrofísica de Andalucía (SEV-2017-0709). A.d.O. acknowledges financial support from the Spanish MCI through Grant PID2019-106027GB-C41. J.P. acknowledges financial support from the Spanish MCI through Grant PID2019-106027GB-C43. S.P. was partially supported by the Polish Funding Agency National Science Centre, project 2017/26/-A/ST9/-00756 (MAESTRO 9).

Acknowledgments: Photoionization computations were mostly done on the departmental server hypercat at IAA-CSIC, on monster at the Department of Physics and Astronomy Galileo Galilei of the University of Padova, and on the institute server "chuck" at CAMK-Warsaw.

Conflicts of Interest: The authors declare no conflict of interest.

\section{Abbreviations}

The following abbreviations are used in this manuscript: 


$\begin{array}{ll}\text { AGN } & \text { active galactic nucleus } \\ \text { BLR } & \text { broad line region } \\ \text { EC } & \text { Electronic configuration } \\ \text { FIZ } & \text { fully ionized zone } \\ \text { FWHM } & \text { full-width half-maximum } \\ \text { HIL } & \text { high-ionization line } \\ \text { IIL } & \text { intermediate-ionization line } \\ \text { LIL } & \text { low-ionization line } \\ \text { MS } & \text { main sequence } \\ \text { NLSy1 } & \text { Narrow-Line Seyfert 1 } \\ \text { PIZ } & \text { partially ionized zone }\end{array}$

\section{Appendix A. Electron-Ion Collisions}

Electron-ion collisions are expected to be the dominant contributor to the emission of optically thick resonance lines. Collisions in a plasma with free electrons and positive ions could excite the ion in initial state $i$ to a higher state $j$. The excited state $j$ decays by the emission of a photon $h v_{\mathrm{ij}}$, producing an emission line.

$$
e^{-}+X_{i}^{+n} \rightarrow e^{-}+X_{j}^{+n} ; X_{j}^{+n} \rightarrow X_{i}^{+n}+h v_{\mathrm{ij}}
$$

This mechanism is the dominant contributor to the emission of forbidden lines, although resonant emission lines are also reputed to be predominantly emitted via collisional excitation, since the initial state is the ground state.

The collisional excitation rate $q_{\mathrm{ij}}[3]$ is given by:

$$
q_{\mathrm{ij}}=\frac{\beta}{\sqrt{T}} \frac{Y_{\mathrm{ij}}}{g_{\mathrm{i}}} \exp \left(-\frac{\epsilon_{\mathrm{ij}}}{k T}\right)
$$

where $g_{\mathrm{i}}$ is the statistical weight of the lower level, $\mathrm{Y}_{\mathrm{ij}}$ the effective collision strength, $T_{\mathrm{e}}$ the electron temperature, $\epsilon_{l u}$ the energy level difference, and $\beta$ a constant. The effective collision strength can be written as:

$$
Y_{\mathrm{ij}}(T)=\int_{0}^{\infty} \Omega_{\mathrm{ij}}(\epsilon) \exp \left(-\frac{\epsilon}{k T}\right) d\left(\frac{\epsilon}{k T}\right)
$$

where the collision strength $\Omega_{\mathrm{ij}}$ has been weighted over the distribution of electron energies, assumed to be Maxwellian at a given electron temperature. The values of $Y_{i j}$ were reported in [3,59] or in the extensive database CHIANTI used in the photoionization calculations [60,61].

\section{References}

1. Netzer, H. AGN emission lines. In Active Galactic Nuclei; Blandford, R.D., Netzer, H., Woltjer, L., Courvoisier, T.J.-L., Mayor, M., Eds.; Springer: Berlin, Germany, 1990; pp. 57-160.

2. Peterson, B.M. An Introduction to Active Galactic Nuclei; Cambridge University Press: Cambridge, UK, 1997.

3. Osterbrock, D.E.; Ferland, G.J. Astrophysics of Gaseous Nebulae and Active Galactic Nuclei; University Science Books: Mill Valley, CA, USA, 2006.

4. Boroson, T.A.; Green, R.F. The emission-line properties of low-redshift quasi-stellar objects. Astrophys. J. Suppl. Ser. 1992, 80, 109-135. [CrossRef]

5. Sulentic, J.W.; Marziani, P.; Dultzin-Hacyan, D. Phenomenology of Broad Emission Lines in Active Galactic Nuclei. Annu. Rev. Astron. Astrophys. 2000, 38, 521-571. [CrossRef]

6. Shen, Y.; Ho, L.C. The diversity of quasars unified by accretion and orientation. Nature 2014, 513, $210-213$. [CrossRef] [PubMed]

7. Marziani, P.; Sulentic, J.W.; Negrete, C.A.; Dultzin, D.; Zamfir, S.; Bachev, R. Broad-line region physical conditions along the quasar eigenvector 1 sequence. Mon. Not. R. Astron. Soc. 2010, 409, 1033-1048. [CrossRef]

8. Panda, S.; Czerny, B.; Adhikari, T.P.; Hryniewicz, K.; Wildy, C.; Kuraszkiewicz, J.; Śniegowska, M. Modeling of the Quasar Main Sequence in the Optical Plane. Astrophys. J. 2018, 866, 115. [CrossRef] 
9. Panda, S.; Marziani, P.; Czerny, B. The Quasar Main Sequence explained by the combination of Eddington ratio, metallicity and orientation. arXiv 2019, arXiv:1905.01729.

10. Fraix-Burnet, D.; Marziani, P.; D'Onofrio, M.; Dultzin, D. The Phylogeny of Quasars and the Ontogeny of Their Central Black Holes. Front. Astron. Space Sci. 2017, 4, 1. [CrossRef]

11. Sulentic, J.W.; Marziani, P.; Zwitter, T.; Dultzin-Hacyan, D.; Calvani, M. The Demise of the Classical Broad-Line Region in the Luminous Quasar PG 1416-129. Astrophys. J. Lett. 2000, 545, L15-L18. [CrossRef]

12. Sulentic, J.W.; Bachev, R.; Marziani, P.; Negrete, C.A.; Dultzin, D. C IV $\lambda 1549$ as an Eigenvector 1 Parameter for Active Galactic Nuclei. Astrophys. J. 2007, 666, 757-777. [CrossRef]

13. Richards, G.T.; Kruczek, N.E.; Gallagher, S.C.; Hall, P.B.; Hewett, P.C.; Leighly, K.M.; Deo, R.P.; Kratzer, R.M.; Shen, Y. Unification of Luminous Type 1 Quasars through C IV Emission. Astrophys. J. 2011, 141, 167. [CrossRef]

14. Coatman, L.; Hewett, P.C.; Banerji, M.; Richards, G.T. C iv emission-line properties and systematic trends in quasar black hole mass estimates. Mon. Not. R. Astron. Soc. 2016, 461, 647-665. [CrossRef]

15. Sulentic, J.W.; del Olmo, A.; Marziani, P.; Martínez-Carballo, M.A.; D’Onofrio, M.; Dultzin, D.; Perea, J.; Martínez-Aldama, M.L.; Negrete, C.A.; Stirpe, G.M.; et al. What does Civ $\lambda 1549$ tell us about the physical driver of the Eigenvector Quasar Sequence? Astron. Astrophys. 2017, 608, A122. [CrossRef]

16. Vietri, G.; Piconcelli, E.; Bischetti, M.; Duras, F.; Martocchia, S.; Bongiorno, A.; Marconi, A.; Zappacosta, L.; Bisogni, S.; Bruni, G.; et al. The WISSH Quasars Project IV. BLR versus kpc-scale winds. arXiv 2018, arXiv:1802.03423.

17. Leighly, K.M. Hubble Space Telescope STIS Ultraviolet Spectral Evidence of Outflow in Extreme Narrow-Line Seyfert 1 Galaxies. II. Modeling and Interpretation. Astrophys. J. 2004, 611, 125-152. [CrossRef]

18. Negrete, A.; Dultzin, D.; Marziani, P.; Sulentic, J. BLR Physical Conditions in Extreme Population A Quasars: A Method to Estimate Central Black Hole Mass at High Redshift. Astrophys. J. 2012, 757, 62. [CrossRef]

19. Marinello, A.O.M.; Rodriguez-Ardila, A.; Garcia-Rissmann, A.; Sigut, T.A.A.; Pradhan, A.K. The FeII emission in active galactic nuclei: excitation mechanisms and location of the emitting region. Astrophys. J. 2016, 820, 116. [CrossRef]

20. Peterson, B.M.; Ferrarese, L.; Gilbert, K.M.; Kaspi, S.; Malkan, M.A.; Maoz, D.; Merritt, D.; Netzer, H.; Onken, C.A.; Pogge, R.W.; et al. Central Masses and Broad-Line Region Sizes of Active Galactic Nuclei. II. A Homogeneous Analysis of a Large Reverberation-Mapping Database. Astrophys. J. 2004, 613, 682-699. [CrossRef]

21. Peterson, B.M. Space Telescope and Optical Reverberation Mapping Project: A Leap Forward in Reverberation Mapping. IAU Symp. 2017, 324, 215-218. [CrossRef]

22. Collin-Souffrin, S.; Dumont, A.M. Emission spectra of weakly photoionized media in active nuclei of galaxies. Astron. Astrophys. 1989, 213, 29-48.

23. Collin, S.; Joly, M. The Fe II problem in NLS1s. New Astron. Rev. 2000, 44, 531-537. [CrossRef]

24. Panda, S.; Czerny, B.; Wildy, C.; Śniegowska, M. What Drives the Quasar Main Sequence? Bajan, K., Biernacka, M., Pollo, A., Eds.; Introduction to Cosmology, 3rd Cosmology School: Cracow, Poland, 2019; Volume 9, pp. 199-202.

25. Negrete, C.A.; Dultzin, D.; Marziani, P.; Sulentic, J.W. A New Method to Obtain the Broad Line Region Size of High Redshift Quasars. Astrophys. J. 2014, 794, 95. [CrossRef]

26. Wills, D.; Netzer, H. The 1400 A emission feature in quasi-stellar objects. Astrophys. J. 1979, $233,1-4$. [CrossRef]

27. Zheng, W. The Critical Densities for Some Emission Lines. Astrophys. Lett. Commun. 1988, $27,275$.

28. Feldman, U. Transitions from Metastable Levels Emitted during Short-Duration Bursts: How Valid Are Their Calculated Intensities? Astrophys. J. 1992, 385, 758. [CrossRef]

29. Fine, S.; Croom, S.M.; Bland-Hawthorn, J.; Pimbblet, K.A.; Ross, N.P.; Schneider, D.P.; Shanks, T. The CIV linewidth distribution for quasars and its implications for broad-line region dynamics and virial mass estimation. Mon. Not. R. Astron. Soc. 2010, 409, 591-610. [CrossRef]

30. Mathews, W.G.; Ferland, G.J. What heats the hot phase in active nuclei? Astrophys. J. 1987, 323, $456-467$. [CrossRef]

31. Ferland, G.J.; Done, C.; Jin, C.; Landt, H.; Ward, M.J. State-of-the-art AGN SEDs for photoionization models: BLR predictions confront the observations. Mon. Not. R. Astron. Soc. 2020, 494, 5917-5922. [CrossRef]

32. Ferland, G.J.; Porter, R.L.; van Hoof, P.A.M.; Williams, R.J.R.; Abel, N.P.; Lykins, M.L.; Shaw, G.; Henney, W.J.; Stancil, P.C. The 2013 Release of Cloudy. Rev. Mex. Astron. Astrofísica 2013, 49, 137-163. 
33. Ferland, G.J.; Chatzikos, M.; Guzmán, F.; Lykins, M.L.; van Hoof, P.A.M.; Williams, R.J.R.; Abel, N.P.; Badnell, N.R.; Keenan, F.P.; Porter, R.L.; et al. The 2017 Release Cloudy. Rev. Mex. Astron. Astrofísica 2017, 53, 385-438.

34. Shin, J.; Woo, J.H.; Nagao, T.; Kim, S.C. The Chemical Properties of Low-redshift QSOs. Astrophys. J. 2013, 763, 58. [CrossRef]

35. Nagao, T.; Maiolino, R.; Marconi, A. Gas metallicity in the narrow-line regions of high-redshift active galactic nuclei. Astron. Astrophys. 2006, 447, 863-876. [CrossRef]

36. Śniegowska, M.; Marziani, P.; Czerny, B.; Panda, S.; Martínez-Aldama, M.L.; del Olmo, A.; D’Onofrio, M. High metal content of highly accreting quasars. arXiv 2020, arXiv:2009.14177.

37. Nagao, T.; Marconi, A.; Maiolino, R. The evolution of the broad-line region among SDSS quasars. Astron. Astrophys. 2006, 447, 157-172. [CrossRef]

38. Juarez, Y.; Maiolino, R.; Mujica, R.; Pedani, M.; Marinoni, S.; Nagao, T.; Marconi, A.; Oliva, E. The metallicity of the most distant quasars. Astron. Astrophys. 2009, 494, L25-L28. [CrossRef]

39. Sulentic, J.W.; Marziani, P.; del Olmo, A.; Dultzin, D.; Perea, J.; Alenka Negrete, C. GTC spectra of z $\approx 2.3$ quasars: comparison with local luminosity analogs. Astron. Astrophys. 2014, 570, A96. [CrossRef]

40. Negrete, C.A.; Dultzin, D.; Marziani, P.; Sulentic, J.W. Reverberation and Photoionization Estimates of the Broad-line Region Radius in Low-z Quasars. Astrophys. J. 2013, 771, 31. [CrossRef]

41. Martinez-Aldama, M.L.; Del Olmo, A.; Marziani, P.; Sulentic, J.W.; Negrete, C.A.; Dultzin, D.; Perea, J.; D’Onofrio, M. Highly Accreting Quasars at High Redshift. Front. Astron. Space Sci. 2018, 4, 65. [CrossRef]

42. Bachev, R.; Marziani, P.; Sulentic, J.W.; Zamanov, R.; Calvani, M.; Dultzin-Hacyan, D. Average Ultraviolet Quasar Spectra in the Context of Eigenvector 1: A Baldwin Effect Governed by the Eddington Ratio? Astrophys. J. 2004, 617, 171-183. [CrossRef]

43. Sulentic, J.; Marziani, P.; Zamfir, S. The Case for Two Quasar Populations. Balt. Astron. 2011, $20,427-434$. [CrossRef]

44. Wang, J.M.; Qiu, J.; Du, P.; Ho, L.C. Self-shadowing Effects of Slim Accretion Disks in Active Galactic Nuclei: The Diverse Appearance of the Broad-line Region. Astrophys. J. 2014, 797, 65. [CrossRef]

45. Hamann, F.; Ferland, G. Elemental Abundances in Quasistellar Objects: Star Formation and Galactic Nuclear Evolution at High Redshifts. Annu. Rev. Astron. Astrophys. 1999, 37, 487-531. [CrossRef]

46. Cano-Díaz, M.; Maiolino, R.; Marconi, A.; Netzer, H.; Shemmer, O.; Cresci, G. Observational evidence of quasar feedback quenching star formation at high redshift. Astron. Astrophys. 2012, 537, L8. [CrossRef]

47. Hamann, F.; Ferland, G. The Chemical Evolution of QSOs and the Implications for Cosmology and Galaxy Formation. Astrophys. J. 1993, 418, 11. [CrossRef]

48. Nagao, T.; Maiolino, R.; Marconi, A.; Matsuoka, K.; Taniguchi, Y. Metallicity Evolution of AGNs; Peterson, B.M., Somerville, R.S., Storchi-Bergmann, T., Eds.; Cambridge University Press: Cambridge, UK, 2010; Volume 267, pp. 73-79. [CrossRef]

49. Gibson, R.R.; Jiang, L.; Brandt, W.N.; Hall, P.B.; Shen, Y.; Wu, J.; Anderson, S.F.; Schneider, D.P.; Vanden Berk, D.; Gallagher, S.C.; et al. A Catalog of Broad Absorption Line Quasars in Sloan Digital Sky Survey Data Release 5. Astrophys. J. 2009, 692, 758-777. [CrossRef]

50. Dultzin, D.; Marziani, P.; de Diego, J.A.; Negrete, C.A.; Del Olmo, A.; Martínez-Aldama, M.L.; D'Onofrio, M.; Bon, E.; Bon, N.; Stirpe, G.M. Extreme quasars as distance indicators in cosmology. Front. Astron. Space Sci. 2020, 6, 80. [CrossRef]

51. Marziani, P.; Sulentic, J.W.; Negrete, C.A.; Dultzin, D.; Del Olmo, A.; Martínez Carballo, M.A.; Zwitter, T.; Bachev, R. UV spectral diagnostics for low redshift quasars: estimating physical conditions and radius of the broad line region. Astrophys. Space Sci. 2015, 356, 339-346. [CrossRef]

52. Laor, A.; Jannuzi, B.T.; Green, R.F.; Boroson, T.A. The Ultraviolet Properties of the Narrow-Line Quasar I ZW 1. Astrophys. J. 1997, 489, 656. [CrossRef]

53. Panda, S.; Martínez-Aldama, M.L.; Marinello, M.; Czerny, B.; Marziani, P.; Dultzin, D. Optical Fe II and Near-Infrared Ca II triplet emission in active galaxies: (I) Photoionization modeling. arXiv 2020, arXiv:2004.05201.

54. Temple, M.J.; Ferland, G.J.; Rankine, A.L.; Hewett, P.C.; Badnell, N.R.; Ballance, C.P.; Del Zanna, G.; Dufresne, R.P. Fe III emission in quasars: evidence for a dense turbulent medium. Mon. Not. R. Astron. Soc. 2020, 496, 2565-2576. [CrossRef] 
55. Hayes, M.A.; Nussbaumer, H. The O IV infrared and ultraviolet flux ratios as temperature and density diagnostics. Astron. Astrophys. 1983, 124, 279-282.

56. Osterbrock, D.E. Expected ultraviolet emission spectrum of a gaseous nebula. Planet. Space Sci. 1963, 11, 621-632. [CrossRef]

57. Padovani, P.; Rafanelli, P. Mass-luminosity relationships and accretion rates for Seyfert 1 galaxies and quasars. Astron. Astrophys. 1988, 205, 53-70.

58. King, A.; Pounds, K. Powerful Outflows and Feedback from Active Galactic Nuclei. Annu. Rev. Astron. Astrophys. 2015, 53, 115-154. [CrossRef]

59. Pradhan, A.K.; Nahar, S.N. Atomic Astrophysics and Spectroscopy; Cambridge University Press: Cambridge, UK, 2015.

60. Dere, K.P.; Del Zanna, G.; Young, P.R.; Landi, E.; Sutherland, R.S. CHIANTI-An Atomic Database for Emission Lines. XV. Version 9, Improvements for the X-ray Satellite Lines. Astrophys. J. Suppl. Ser. 2019, 241, 22. [CrossRef]

61. Del Zanna, G.; Dere, K.P.; Young, P.R.; Landi, E.; Mason, H.E. CHIANTI-An atomic database for emission lines. Version 8. Astron. Astrophys. 2015, 582, A56. [CrossRef]

Publisher's Note: MDPI stays neutral with regard to jurisdictional claims in published maps and institutional affiliations.

(C) 2020 by the authors. Licensee MDPI, Basel, Switzerland. This article is an open access article distributed under the terms and conditions of the Creative Commons Attribution (CC BY) license (http:// creativecommons.org/licenses/by/4.0/). 